\title{
Letters to the Editor-in-Chief
}

\section{Re: "Cost Shifting and Timeliness of Drug Formulary Recommendations in Atlantic Canada," Healthcare Policy, Vol. 5, No. 3, 2010}

May 20, 2010

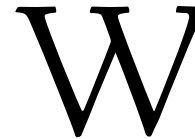

E Wish to COMmend the authors of the above article on taCKling an interesting subject. We would also like to address some inaccuracies regarding CADTH's Common Drug Review (CDR) program and note some recent changes.

The article misleadingly indicates in several places that CDR's response time in reviewing drugs and issuing recommendations "did not improve" or "increased" over the study period.

In fact, CDR has consistently met the aggressive, targeted review time frames that were established prior to the program's launch in 2003. Adherence to these established timelines is a critical component of the CDR process that we take very seriously - as it ultimately affects patient access to medications.

All drugs reviewed by CDR have met the meeting date of the Canadian Expert Drug Advisory Committee (CEDAC) for which they were targeted, or an earlier CEDAC meeting date. That is, all reviews have met or bettered the established 19- to 25-week time frame for CDR review and CEDAC recommendation.

The drug reviews noted in the article that extended beyond 25 weeks were generally conducted within the established timelines for the additional steps that were involved in those particular reviews. Specifically, each of those reviews involved one, if not more, of the following steps:

- a Request for Extension of the Embargo Period by the drug manufacturer

- a Request for Reconsideration by the drug manufacturer

- a Request for Clarification by the participating drug plans

- deferral of the recommendation by CEDAC (so that further information could be gathered by the CDR Directorate).

Additionally, CADTH has made numerous improvements to the CDR process since program inception. Several of these enhancements were specifically designed to reduce $C D R$ review timelines or reduce the time to formulary listing post-Notice of Compliance. For instance:

- "Resubmissions based on a reduced price during the 10-day embargo period" 
enable manufacturers to resubmit without being placed at the end of the review queue with other resubmissions (effective April 20, 2009).

- CDR priority review refinements give drug manufacturers an option to reduce the time for manufacturer's comments and reviewers' replies - so that the drug may be placed on an earlier CEDAC agenda.

- "Pre-NOC priority review submissions" enable manufacturers to file submissions with the CDR Directorate before receiving a Health Canada Notice of Compliance (NOC) or Notice of Compliance with conditions (effective July 1, 2009).

One final clarification: the study claims its findings "uphold the criticism that CDR has not reduced variation among provincial formularies."

In fact, the opposite is true. Our calculations, based on publicly available information, indicate that the decisions of the participating drug plans have followed the CDR recommendations more than $90 \%$ of the time.

This is despite the fact that, in addition to the CDR recommendation, the drug plan decisions must take into account individual drug plan mandates, jurisdictional priorities and financial resources.

Given that the CDR is still a relatively new program and has only issued recommendations for a small proportion of the drugs on the jurisdictional formularies at this point, one would reasonably expect that the full effect of CDR's centralized review and recommendation process, in terms of increased consistency amongst formularies, will be realized over a longer time horizon.

Sincerely,

Sandra Pagotto

Senior Director, Common Drug Review, Canadian Agency for Drugs and Technologies in Health

\section{The Author's Reply}

June 22, 2010

I HANK YOU FOR THE OPPORTUNITY TO RESPOND TO THE LETTER FROM the Canadian Agency for Drugs and Technologies in Health (CADTH) on

1 May 20th. While we welcome an open dialogue concerning the results of our study, we wish to clarify some of the concerns brought up by Ms. Pagotto.

Firstly, regarding the issue that we misleadingly indicated that the CDR response time in our manuscript increased, you will note that it is just for the 35 selected drugs we reviewed during the time period of January 1, 2005, to May 1, 2008. This is clearly 
stated on page 105. The drugs we reviewed can be found listed in Table 1 along with their respective review times. It is clearly shown that for the drugs reviewed in our study the timeline for CDR review did increase. You will also find on page 111 that we acknowledge CADTH's time frame of 19-25 weeks for review of drug submissions. Our argument is to encourage adherence to this time frame, and we agree with Ms. Pagotto that it is critical to maintaining the success of the CDR process. With regard to Ms. Pagotto's argument that the drug reviews that extended beyond 25 weeks required additional steps, we acknowledged this issue on page 111 as well, indicating that the CDR does not have control over the quality of submissions it receives and whether additional information is required. However, our aim was to measure the overall average review time during the three-year study period for the 35 drugs included in the study, which clearly and objectively showed an overall increase in CDR review time. The improvements to the CDR process listed by Ms. Pagotto were not applicable to any of the 35 drugs included in the study.

Secondly, regarding our claim that the study findings uphold criticism that the CDR has not reduced variation among provincial formularies, we stand behind that criticism based upon the findings from the 35 drugs included during the study period. Table 2 demonstrates our findings that timeliness of uptake of CDR-recommended drugs not only varied by year within each of the four Atlantic Provinces, it also varied considerably between all four provinces. We acknowledge on page 107 that timeliness in both New Brunswick and PEI improved over the study period; however, it did not improve in Nova Scotia and Newfoundland. Additionally, PEI had failed to adopt two CDR recommended drugs by the end of the study. Similar results and arguments have been previously presented in literature (Grootendorst 2002; Marra et al. 2006; McMahon et al. 2006). While Ms. Pagotto argues that the decisions of participating drug plans follow CDR recommendations more than $90 \%$ of the time, this does not speak to variations in the timeliness of uptake of CDR recommendations, which was the objective of this paper. We acknowledge that variation in listings may occur due to financial restraints and capacity in each province (page 111) as Ms. Pagotto points out; however, we argue that participating drug plans should renew their commitment to the CDR process to decrease variation and increase transparency when delays do occur.

Overall, we feel the arguments put forth in our paper are upheld by the findings of the study and do not misrepresent the efforts of CADTH, the CDR or participating drug plans. We hope that you find this response helpful. Please let us know if you require any further clarification.

Sincerely,

Andrea Scobie, MHSA

Dalhousie University, College of Pharmacy 


\section{REFERENCES}

Grootendorst. 2002. "Beneficiary Cost Sharing Under Canadian Provincial Prescription Drug Benefit Programs: History and Assessment." PharmacoEconomics 9(2): 79-99.

Marra, C.A., L.D. Lynd, A.H. Anis and J.M. Esdaile. 2006. "Approval Process and Access to Prescription Drugs in Canada." Arthritis Care E Research 55(1): 9-11.

McMahon, M., S. Morgan and C. Mitton. 2006."The Common Drug Review: A NICE Start for Canada?" Health Policy 77(3): 339-51.

\begin{tabular}{l|l|l} 
UNIVERSITY & Faculty of \\
\hline Community Health Sciences
\end{tabular}

\section{COMING SOON:}

Healthcare Policy presents a special issue of proceedings from the Manitoba Centre for Health Policy 20th Anniversary Conference.

\section{Featuring summaries of keynote presentations by:}

Dr. Clyde Hertzman, "The Role of

Administrative Record Linkage in Creating

Trajectories of Early Human Development"

Dr. Fiona Stanley, "The Developmental

Pathways Project: Can Joined-Up Data Lead to Joined-Up Thinking?"

Mr. Steven Lewis, "How Health Services

Research has Made a Difference"

Dr. Patricia Martens, “Going for Knowledge

Translation Gold: Research with its Feet on the Ground...Walking and Talking" 